\title{
Particle detectors, geodesic motion, and the equivalence principle
}

\author{
Sebastiano Sonego* and Hans Westman ${ }^{\dagger}$ \\ *Università di Udine, Via delle Scienze 208, 33100 Udine, Italy \\ ${ }^{\dagger}$ Department of Astronomy and Astrophysics, \\ Chalmers University of Technology, 41296 Göteborg, Sweden
}

October 29, 2003; LTEX-ed October 29, 2018

\begin{abstract}
It is shown that quantum particle detectors are not reliable probes of spacetime structure. In particular, they fail to distinguish between inertial and non-inertial motion in a general spacetime. To prove this, we consider detectors undergoing circular motion in an arbitrary static spherically symmetric spacetime, and give a necessary and sufficient condition for the response function to vanish when the field is in the static vacuum state. By examining two particular cases, we show that there is no relation, in general, between the vanishing of the response function and the fact that the detector motion is, or is not, geodesic. In static asymptotically flat spacetimes, however, all rotating detectors are excited in the static vacuum. Thus, in this particular case the static vacuum appears to be associated with a non-rotating frame. The implications of these results for the equivalence principle are considered. In particular, we discuss how to properly formulate the principle for particle detectors, and show that it is satisfied.
\end{abstract}

PACS: 04.62.+v; 04.20.Cv

Keywords: Unruh effect; vacuum; equivalence principle.

*sebastiano.sonego@uniud.it

${ }^{\dagger}$ hawe@fy.chalmers.se 


\section{Introduction}

A particle detector in Minkowski spacetime remains unexcited when the field is in the vacuum state, if it moves along a geodesic, i.e., along a straight line in space at a constant speed [1]. This property is often regarded as a quantum counterpart of the law of inertia quantum detectors and classical mechanics experiments identify the same class of preferred reference frames ${ }^{1}[2$. By a straightforward application of the equivalence principle, one would then expect that in an arbitrary spacetime, a detector in geodesic motion should not become excited, provided the field is in the vacuum state. However, this statement, as well as its converse - that a non-inertial detector in an arbitrary spacetime is somewhat analogous to an accelerated detector in Minkowski spacetime, and should therefore get excited $^{2}$-, is trivially false, unless formulated more precisely. Indeed, in a non-stationary spacetime there is no meaningful notion of a "vacuum state", because particle creation takes place [1]. In other cases one can have inequivalent vacua [3, 4, and the opposite problem arises. This happens already in Minkowski spacetime, where a uniformly accelerated particle detector in the so-called Rindler vacuum does not become excited, although it does so in the usual Minkowski vacuum [1, 5. Then, the notion of a vacuum becomes ambiguous.

Nevertheless, one may define a vacuum state directly in terms of detectors response, considering their behaviour in all possible frames, for each possible state of the field. If there happens to be a state, for which there exists at least one reference frame in which the detectors carried by all observers are not excited, then one can identify it with a vacuum. Conversely, knowing in advance the vacuum state, one can find the frames in which detectors do not get excited. Thus, a null response can be used in two different ways, in order to identify both a vacuum state and one or more preferred frames associated with it. ${ }^{3}$

We can now reformulate the statement above in a more precise way. Suppose that in a given spacetime one can find a state of the field in which detectors carried by one particular geodesic frame are not excited. Then, one expects that the same should happen for all geodesic frames, and only for them. One aim of the present note is to disprove this claim by exhibiting two counterexamples. In doing so, we shall also show that quantum detectors are not reliable probes of spacetime structure. This fact, in turn, will help elucidating the status of the equivalence principle in quantum field theory.

Our strategy is based on the fact that, in a static spacetime, one can define a state in which static quantum detectors are not excited — a static vacuum. Of course, in general this has nothing to do with the state in which an inertial detector does not become excited. However, in the particular case of a so-called ultrastatic spacetime [7, a static frame is also geodesic. It is then sufficient to find ultrastatic spacetimes such that, when the field is in a static vacuum, either there are non-geodesic detectors which also do not get excited,

\footnotetext{
${ }^{1}$ Hereafter, by observer we mean a future-directed, differentiable timelike curve in spacetime. A reference frame is a congruence of observers.

${ }^{2}$ This is often used as a heuristic explanation for particle creation by a gravitational field.

${ }^{3}$ This operational definition of a vacuum is not directly equivalent, in general, to the one based on canonical field quantisation [4]. See, however, reference 6] for an attempt to reconcile the two definitions, based on taking into account the spurious effects due to the detector structure. The operationalistic approach could be criticised by noticing that "particles" are not fundamental objects in quantum field theory, which is in fact about fields.
} 
or there are geodesic detectors which do get excited. Taken together, these two types of behaviour show that there is no correlation whatsoever between the response of a detector and the fact that its motion is, or is not, geodesic.

The article is organised as follows. We restrict our attention to the case of static spherically symmetric spacetimes. In the next section we define a static vacuum and present some background calculations about the response function of a quantum detector that moves on a circular orbit. ${ }^{4}$ In section 3 we establish a necessary and sufficient condition for the response function to vanish in the static vacuum. In section 4 we show that in a static, asymptotically flat spacetime, rotating detectors do not become excited when the field is in a static vacuum iff their worldlines coincide with those of the static observers. Hence, in this case detectors identify the same state of non-rotation of classical mechanical experiments. In general, however, they fail to do so. This happens, e.g., in Einstein's static universe, where there are non-geodesic motions for which the no-detection condition is satisfied, as shown in section [5.1. Conversely, in section [5.2 we describe a class of spacetimes for which the condition is violated by detectors that move freely along circular orbits - hence, with geodesic worldlines. Section [6] contains a discussion about the validity of the equivalence principle in quantum field theory, and some concluding remarks.

\section{Preliminaries}

In the following, we consider a real scalar field $\phi$, obeying the field equation ${ }^{5}$

$$
g^{a b} \nabla_{a} \nabla_{b} \phi-\xi R \phi-\mu^{2} \phi=0
$$

in a spacetime $\left(\mathcal{M}, g_{a b}\right)$, where $\xi$ and $\mu$ are constants and $R$ is the Ricci curvature scalar.

\subsection{Vacuum state}

Let $f_{I}$ be positive-norm functions that, together with their complex conjugates $f_{I}^{*}$, form a complete set in the space of solutions of (2.1), and let $|0\rangle$ be the state such that $\hat{a}_{I}|0\rangle=0$, where the annihilation operators $\hat{a}_{I}$ are defined through the decomposition

$$
\hat{\phi}(x)=\sum_{I}\left(f_{I}(x) \hat{a}_{I}+f_{I}(x)^{*} \hat{a}_{I}^{\dagger}\right)
$$

of the field operator $\hat{\phi}$. Then, the response function of a DeWitt monopole detector for $\phi$ in the state $|0\rangle$ is [1]

$$
\mathcal{R}(E ; \gamma)=\lim _{T \rightarrow+\infty} \frac{\Theta(E)}{2 T} \int_{-T}^{T} \mathrm{~d} \tau \int_{-T}^{T} \mathrm{~d} \tau^{\prime} \mathrm{e}^{-\mathrm{i} E\left(\tau-\tau^{\prime}\right)} G^{+}\left(x(\tau), x\left(\tau^{\prime}\right)\right)
$$

\footnotetext{
${ }^{4}$ The response of rotating detectors was also considered in other papers for different purposes than ours. Such previous analysis have been performed in order to gain a deeper understanding of the detector response [6, 8], to investigate the properties of alternative vacuum states [9] [0], and to explore the possibility for experimental tests of Unruh-like effects [11.

${ }^{5}$ We work in units with $c=\hbar=1$, and choose the metric signature to be +2 . Tensor indices from the beginning of the Latin alphabet, $a, b, c, \ldots$, run from 0 to 3 . The conventions for the curvature tensors are the same adopted in reference [12].
} 
Here $\gamma$ denotes the detector worldline, expressed by the coordinate functions $x^{a}(\tau)$ of the detector proper time $\tau, \Theta$ is the step function, and

$$
G^{+}\left(x, x^{\prime}\right)=\left\langle 0\left|\hat{\phi}(x) \hat{\phi}\left(x^{\prime}\right)\right| 0\right\rangle=\sum_{I} f_{I}(x) f_{I}\left(x^{\prime}\right)^{*}
$$

is the Wightman function. Physically, $\mathcal{R}(E ; \gamma)$ is the probability per unit time that the detector be excited from its ground state to the energy eigenstate with energy $E$. This transition is usually regarded as corresponding to the detection of a quantum of the field with energy $E$, although such an interpretation is not free from ambiguities [13. If the modes $f_{I}$ have positive frequency with respect to $\gamma$, i.e., if

$$
\int_{-\infty}^{+\infty} \mathrm{d} \tau \mathrm{e}^{-\mathrm{i} E \tau} f_{I}(x(\tau))
$$

is non-zero only for negative values of $E$, then $\mathcal{R}(E ; \gamma)=0$ and $|0\rangle$ is recognised as a vacuum state according to the operational definition given in section 1 .

\subsection{Modes and frequency spectrum}

We work in a static, spherically symmetric spacetime with the metric

$$
g=-A(\rho)^{2} \mathrm{~d} t^{2}+\mathrm{d} \rho^{2}+r(\rho)^{2}\left(\mathrm{~d} \theta^{2}+\sin ^{2} \theta \mathrm{d} \varphi^{2}\right)
$$

where $A$ and $r$ are two positive functions, that we leave unspecified for the moment. A convenient set of positive-norm solutions of the field equation (2.1) is then given by modes of the form

$$
f_{l m}^{(\sigma)}(\rho, \theta, \varphi \mid \omega) \mathrm{e}^{-\mathrm{i} \omega t}
$$

with $\omega>0$. In general, $\omega$ belongs to a spectrum which has both a discrete and a continuous part, and the label $\sigma$ accounts for a possible degeneracy of the parameters $\omega, l$, and $m$ [14. These are positive-frequency modes with respect to static observers. Hence, the corresponding vacuum state is such that static detectors register no particles. We shall refer to this as the static vacuum (also known as the "Boulware vacuum" [15]).

Because of spherical symmetry, we can separate variables and write straightforwardly

$$
f_{l m}^{(\sigma)}(\rho, \theta, \varphi \mid \omega)=\frac{(-1)^{m}}{r} \chi_{l}^{(\sigma)}(\rho \mid \omega)\left(\frac{2 l+1}{4 \pi} \frac{(l-m) !}{(l+m) !}\right)^{1 / 2} P_{l}^{m}(\cos \theta) \mathrm{e}^{\mathrm{i} m \varphi}
$$

where $P_{l}^{m}$ are the associated Legendre functions and $l, m$ are integer numbers with $l \geq 0$ and $-l \leq m \leq l$ (see, e.g., reference [16]). The functions $\chi_{l}^{(\sigma)}(\rho \mid \omega)$ are then solutions of the differential equation

$$
\frac{\mathrm{d}^{2} \chi_{l}^{(\sigma)}}{\mathrm{d} x^{2}}+\left(\omega^{2}-\frac{1}{r} \frac{\mathrm{d}^{2} r}{\mathrm{~d} x^{2}}-\xi A^{2} R-A^{2} \mu^{2}-A^{2} \frac{l(l+1)}{r^{2}}\right) \chi_{l}^{(\sigma)}=0,
$$

where the variable $x$, defined by

$$
\mathrm{d} x=\frac{1}{A(\rho)} \mathrm{d} \rho
$$


is a generalised Regge-Wheeler "tortoise" coordinate [17]. Formally, (2.9) is a timeindependent one-dimensional Schrödinger equation for a non-relativistic particle of mass $1 / 2$ and energy $\omega^{2}>0$ in an external field with potential energy

$$
V_{l}(x)=\frac{A(\rho)}{r(\rho)} \frac{\mathrm{d}}{\mathrm{d} \rho}\left(A(\rho) \frac{\mathrm{d} r(\rho)}{\mathrm{d} \rho}\right)+\xi A(\rho)^{2} R(\rho)+A(\rho)^{2} \mu^{2}+A(\rho)^{2} \frac{l(l+1)}{r(\rho)^{2}},
$$

where we have transformed the first term on the right hand side using (2.10), and $\rho$ must be regarded as a function of $x$, obtained by integrating (2.10) and inverting the result. Thus, equation (2.9) represents an eigenvalue problem: For an arbitrary value $l \geq 0$, solving (2.9) means not only to find a function $\chi_{l}^{(\sigma)}(\rho \mid \omega)$, but also to determine the spectrum $\mathcal{S}_{l}$ of the frequencies $\omega \in \mathcal{S}_{l}$ compatible with the assigned $l$. The total frequency spectrum is then

$$
\mathcal{S}=\bigcup_{l=0}^{+\infty} \mathcal{S}_{l}
$$

In general, $\mathcal{S}_{l}$ (hence $\mathcal{S}$ ) is the union of a discrete and a continuous part. Qualitatively, the structure of the spectrum $\mathcal{S}_{l}$ can be determined by analysing the potential energy $V_{l}$ given by (2.11), just as one does traditionally in quantum mechanics. Indeed, the possible values $\omega \in \mathcal{S}_{l}$ coincide with the square root of the energy eigenvalues for the timeindependent Schrödinger equation (2.9) $\cdot{ }^{6}$ As it is well-known, values of the total energy corresponding to an unbounded classical one-dimensional motion with potential energy $V_{l}$, belong to the continuous part of the quantum mechanical spectrum. On the other hand, values of the total energy that belong to the discrete part of the spectrum, must necessarily correspond to classically bound motions [18].

\subsection{Response function on circular orbits}

Let us consider detector worldlines of the particular type

$$
\left.\begin{array}{l}
t(\tau)=\alpha \tau \\
\rho(\tau)=\text { const } \\
\theta(\tau)=\pi / 2 \\
\varphi(\tau)=\Omega t(\tau)=\alpha \Omega \tau
\end{array}\right\}
$$

which correspond to uniform circular motions. Here $\Omega$ is a constant, and

$$
\alpha=\left(A^{2}-\Omega^{2} r^{2}\right)^{-1 / 2}
$$

by normalisation of the four-velocity. Thus, the possible values of $\Omega$ for an orbit at a given $\rho$ are constrained by the inequality ${ }^{7}$

$$
|\Omega|<A(\rho) / r(\rho) .
$$

\footnotetext{
${ }^{6}$ Of course, only the non-negative part of the quantum mechanical spectrum leads to real values of $\omega$.

${ }^{7}$ This condition can also be re-expressed as $|\Omega| \widetilde{r}<1$, where $\widetilde{r}=r / A$ is the circumferential radius in the corresponding optical geometry [17]. The parameter $\widetilde{r}$ plays the role of radius of gyration for relativistic dynamics [19.
} 
Substituting the parametric equations (2.13) for the worldline into the modes (2.7), then using equations (2.3) and (2.4) one gets, after performing the integrals in $\tau$ and $\tau^{\prime}$ and taking the limit, the response function for a detector which moves along a circular orbit identified by the parameters $\rho$ and $\Omega$ :

$$
\begin{aligned}
\mathcal{R}(E ; \rho, \Omega)= & \frac{\Theta(E)}{\pi r^{2}} \sum_{\sigma} \sum_{l=0}^{+\infty} \sum_{m=-l}^{l} 2^{m-1}(2 l+1) \frac{(l-m) !}{(l+m) !} \frac{\Gamma\left(\frac{l+m+1}{2}\right)}{\Gamma\left(\frac{l-m}{2}+1\right)} \cos ^{2} \frac{(l+m) \pi}{2} \\
& \int_{0}^{+\infty} \mathrm{d} \omega \lambda_{l}(\omega) \delta(E+\alpha(\rho) \omega-m \alpha(\rho) \Omega)\left|\chi_{l}^{(\sigma)}(\rho \mid \omega)\right|^{2}
\end{aligned}
$$

where we have used the explicit expression for $P_{l}^{m}(0)$ [20]. In this expression, $\lambda_{l}(\omega)$ is a non-negative generalised function which accounts for the frequency spectrum, such that $\mathcal{S}_{l}=\left\{\omega \mid \lambda_{l}(\omega) \neq 0\right\}$. In general, $\lambda_{l}$ is a sum of delta functions (discrete part of the spectrum) and step functions (continuous part).

\section{No-detection condition}

The detector with worldline (2.13) remains unexcited iff $\mathcal{R}(E ; \rho, \Omega)=0$ for all values of $E$. Because of the occurrence of the step function $\Theta(E)$ in equation (2.16), this condition is equivalent to the vanishing of the integral on the right-hand side of (2.16) for all positive $E$. Since all terms of the sum in (2.16) are non-negative, the detector is not excited iff each of them, taken separately, is equal to zero. Neglecting coefficients different from zero, we find that the no-detection condition amounts to asking that

$$
\cos ^{2} \frac{(l+m) \pi}{2} \int_{0}^{+\infty} \mathrm{d} \omega \lambda_{l}(\omega) \delta(E+\alpha(\rho) \omega-m \alpha(\rho) \Omega)\left|\chi_{l}^{(\sigma)}(\rho \mid \omega)\right|^{2}=0
$$

$\forall E>0$, and for all possible combinations of $l, m$, and $\sigma$. The cosine coefficients vanish when $l+m$ is odd, hence (3.1) leads to a non-trivial no-detection condition only for even values of $l+m$. Since the measure function $\lambda_{l}$ is, by definition, non-zero when $\omega \in \mathcal{S}_{l}$, this condition is

$$
\delta(E+\alpha(\rho) \omega-m \alpha(\rho) \Omega)\left|\chi_{l}^{(\sigma)}(\rho \mid \omega)\right|^{2}=0,
$$

$\forall E>0$, and for all possible combinations of $l, \omega \in \mathcal{S}_{l}, m$, and $\sigma$, with $l+m$ even. One may worry about the possibility of having degenerate values of $\rho$, where $\chi_{l}^{(\sigma)}(\rho \mid \omega)=0$ for all possible combinations of $l, \omega \in \mathcal{S}_{l}$, and $\sigma$. However, the set of all possible values of $\rho$ for which this can happen must have zero measure, because one requires completeness of the set of modes. Thus, for almost all $\rho$, the set

$$
\mathcal{P}_{\rho}=\left\{(\omega, l) \mid \omega \in \mathcal{S}_{l}, l \geq 0, \exists \sigma \text { such that } \chi_{l}^{(\sigma)}(\rho \mid \omega) \neq 0\right\}
$$

is nonempty. We conclude that the detector at $\rho$ does not click iff the delta function in (3.2) is zero for all $(\omega, l) \in \mathcal{P}_{\rho}$, i.e., iff

$$
E+\alpha(\rho)(\omega-m \Omega) \neq 0,
$$


$\forall E>0$, for all pairs $(\omega, l) \in \mathcal{P}_{\rho}$, and values of $m$ such that $l+m$ is even. (See also reference 9].) Since $E$ must be positive and $\alpha$ is positive by definition, this condition is equivalent to

$$
m \Omega \leq \omega,
$$

again for all $(\omega, l) \in \mathcal{P}_{\rho}$, and $m$ such that $l+m$ is even. Since $\omega \geq 0$, the case $m=0$ always trivially satisfies (3.5), so one can express the no-detection condition more conveniently as $-\Omega_{\rho}^{-} \leq \Omega \leq \Omega_{\rho}^{+}$, where

$$
\Omega_{\rho}^{-}:=\inf \left\{\omega /(-m) \mid(\omega, l) \in \mathcal{P}_{\rho}, l+m \text { even, } m<0\right\}
$$

and

$$
\Omega_{\rho}^{+}:=\inf \left\{\omega / m \mid(\omega, l) \in \mathcal{P}_{\rho}, l+m \text { even, } m>0\right\} .
$$

It is easy to check that

$$
\Omega_{\rho}^{-}=\Omega_{\rho}^{+}=\Delta_{\rho}:=\inf \left\{\omega / l \mid(\omega, l) \in \mathcal{P}_{\rho}\right\},
$$

so the necessary and sufficient no-detection condition for a specific value of $\rho$ becomes

$$
|\Omega| \leq \Delta_{\rho}
$$

If $\Delta_{\rho}=0$, the no-detection condition reduces to $\Omega=0$ : Only detectors belonging to the non-rotating frame do not get excited. In general, however, there could be an entire band of values of $\Omega$ around $\Omega=0$ for which no detection takes place. The value of $\Delta_{\rho}$, which gives the exact width of this band, can be computed by (3.8), once the structure of the frequency spectrum is known. In particular, for each given value of $\rho$ one must know the possible values of $\omega / l$. This, in turn, requires the knowledge of $\mathcal{S}_{l}$, which is determined by finding the eigenvalues of the Schrödinger operator in (2.9) for the given value of $l$. For a concrete example of this calculation, see section 5.1 below.

\section{Quantum non-rotating frame}

In the particular case of an asymptotically flat spacetime, we have

$$
\begin{gathered}
\lim _{\rho \rightarrow+\infty} r(\rho)=+\infty, \\
\lim _{\rho \rightarrow+\infty} A(\rho)=\lim _{\rho \rightarrow+\infty} r^{\prime}(\rho)=1,
\end{gathered}
$$

and

$$
\lim _{\rho \rightarrow+\infty} A^{\prime}(\rho)=\lim _{\rho \rightarrow+\infty} r^{\prime \prime}(\rho)=\lim _{\rho \rightarrow+\infty} R=0,
$$

where $A^{\prime}, r^{\prime}$, and $r^{\prime \prime}$ are derivatives of the functions $A$ and $r$. It follows that

$$
\lim _{\rho \rightarrow+\infty} V_{l}(\rho)=\mu^{2}
$$

for any value of $l$. This implies that the continuous part $\mathcal{S}_{\text {cont }}$ of the spectrum is $\mathcal{S}_{\text {cont }}=$ ]$\mu,+\infty[$, independent of the value of $l$.

Since $\mathcal{S}$ has a continuous part, there is no constraint on $l$ in correspondence to the values of $\omega$. (This can be understood by the quantum mechanical analogy, where the continuous part of the spectrum corresponds to scattering states, for which every value of $l$ is allowed.) Then, $\inf \{\omega / l\}=0$, because for any given value of $\omega$ in $\mathcal{S}_{\text {cont }}$, there is no upper bound to the possible values of $l$, so one can trivially consider the limit $l \rightarrow+\infty$. Hence, $\Delta_{\rho}=0$, which selects just the static frame. 


\section{Detector response and geodesic motion}

In its roughest version, the claim mentioned in the Introduction - that a particle detector should not get excited in a vacuum state, if and only if its motion is geodesic - is trivially false. Indeed, a static detector does not get excited when the field is in the static vacuum defined in section 2.1, and yet its motion is not geodesic for non-constant $A$. One might see this, however, as a drawback of that particular vacuum state rather than evidence for the incorrectness of the claim. After all, the same happens in Minkowski spacetime, where a uniformly accelerated detector may not get excited, provided the field is in the so-called Rindler vacuum [1, 5].

However, if the spacetime is not just static, but ultrastatic — which in our case simply amounts to having $A \equiv 1$ [7] - , then static observers are also geodesic. Hence, the static vacuum is certainly appropriate, and one might expect that any other geodesic detector, and only a geodesic one, should not get excited in it.

We now exhibit two counter-examples to this claim. Taken together, they show that, in general, there exists no relation between zero/non-zero response function and geodesic/nongeodesic motion. Since, in the next section, we want to relate this result to a quantum version of the equivalence principle, it is important to ensure that no violation of it can take place already at the classical level. In fact, it turns out 21] that, among all possible field equations of the form (2.1), only the one with $\xi=1 / 6$ (the so-called conformal coupling between field and curvature) satisfies Einstein's equivalence principle. ${ }^{8}$ Thus, in this section we shall restrict ourselves to considering a conformally coupled scalar field.

\subsection{Einstein's static universe}

The inequality (3.9) has interesting consequences in spacetimes with finite spatial sections 9]. Consider the conformally coupled scalar field in Einstein's static universe, $A=1$ and $r(\rho)=a \sin (\rho / a)$, where $a$ is a positive constant. In this case, the frequency spectrum is discrete,

$$
\omega_{n}=\frac{1}{a}\left((n+1)^{2}+\mu^{2} a^{2}\right)^{1 / 2}
$$

with $n \geq l$, and the functions $\chi_{l}\left(\rho \mid \omega_{n}\right)$ are proportional to the Gegenbauer polynomials $C_{n-l}^{l+1}(r / a)$ 23]. Then we have $\mathcal{S}_{l}=\left\{\omega_{l}, \omega_{l+1}, \ldots\right\}$, and

$$
\begin{aligned}
\Delta_{\rho} & =\frac{1}{a} \inf \left\{\left((n+1)^{2}+\mu^{2} a^{2}\right)^{1 / 2} / l \mid n=0,1, \ldots ; l=0,1, \ldots, n ; C_{n-l}^{l+1}(r / a) \neq 0\right\} \\
& =\frac{1}{a} \inf \left\{\left((n+1)^{2}+\mu^{2} a^{2}\right)^{1 / 2} / n \mid n=0,1, \ldots ; C_{0}^{n+1}(r / a) \neq 0\right\}=\frac{1}{a}, \quad
\end{aligned}
$$

because $C_{0}^{n+1}(r / a) \equiv 1$. Hence, the no-detection condition turns out to be

$$
|\Omega| a \leq 1
$$

for any value of $\rho$. The same conclusion can be obtained by noticing that the no-detection condition is equivalent to

$$
n|\Omega| \leq \omega_{n}
$$

\footnotetext{
${ }^{8}$ We follow reference [22] for the terminology about the various possible formulations of the principle.
} 
for all values of $n$, so

$$
|\Omega| a \leq\left(\left(1+\frac{1}{n}\right)^{2}+\frac{\mu^{2} a^{2}}{n^{2}}\right)^{1 / 2}
$$

for all $n$. The tighter constraint corresponds to $n \rightarrow+\infty$, and gives again (5.3). Notice that our condition (5.3) is at variance with inequality (16) in reference [9].

Since the detector must travel at a speed smaller than the speed of light, we also have from (2.15) that $|\Omega|<1 / r$. On a great circle $r=a$, this inequality coincides with the no-detection condition (5.3). Therefore, a detector with constant speed on a great circle (and hence in geodesic motion) does not become excited (see also reference 9]). This, at first sight, seems to support the conjecture, because motion at a constant speed along a great circle is geodesic. But consider instead a detector on a circular orbit that is not a great circle, $r<a$. Such an orbit does not correspond to geodesic motion. ${ }^{9}$ The no-detection condition (5.3), however, is still satisfied, provided $|\Omega|$ is smaller than $1 / a$. Thus, for sufficiently small speeds, the detector is not excited, although its motion is nongeodesic. We can also conclude from this result that the detector fails to reveal the state of non-rotation.

Interestingly, all this follows directly from a general theorem due to Chmielowski, who has shown that the vacuum states associated with two global complete commuting timelike Killing vector fields coincide [24. Indeed, in the Einstein static universe, $\partial_{t}$ and $\partial_{t}+\Omega \partial_{\varphi}$ are two such fields, when $\Omega$ is a constant such that $|\Omega|<1 / a$. This implies that detectors with four-velocity parallel to $\partial_{t}+\Omega \partial_{\varphi}$, where $|\Omega|<1 / a$, do not get excited when the field is in the static vacuum, in agreement with the analysis presented above.

\subsection{Asymptotically flat ultrastatic spacetime}

When the function $r(\rho)$ possesses local maxima or minima, there are circular geodesics $\rho=$ const on the spatial sections $t=$ const. These can be easily visualised in terms of the occurrence of throats and bulges in the embedding diagram of equatorial planes [17. Motion at constant speed along these circles corresponds to some particular timelike geodesics in spacetime. From the analysis in section 4 , it follows that in an asymptotically flat spacetime, detectors on these geodesics do get excited in the static vacuum. ${ }^{10}$ From this particular class of examples, one realises that not only detectors do not reveal the spacetime structure; they do not even reveal the geometry of three-dimensional space (either ordinary or optical).

\section{Discussion}

We have presented both an example in which a non-geodesic detector does not become excited (section 5.11), as well as one where a geodesic detector has a non-vanishing response

\footnotetext{
${ }^{9}$ In any ultrastatic spacetime, a spacetime geodesic corresponds to motion along a spatial geodesic at a constant speed.

${ }^{10}$ The "conspiratorial" possibility that the modes $\chi_{l}^{(\sigma)}$ could all vanish just for these values of $\rho$ can be excluded by considering a spacetime in which the bulge, or throat, is actually a finite portion of a flat cylinder. In this case the modes should all vanish within a finite interval of values of $\rho$, which is excluded by completeness.
} 
function (section 5.2). Thus, in general, there is no correlation between the vanishing of the response function of a particle detector in a vacuum state, and the fact that the detector worldline is, or is not, a geodesic in spacetime.

With a little hindsight, it is easy to realise that there really was little reason to believe in the existence of such correlation. The response function does not depend directly on local geometry but on global field modes. That there is a nice coincidence in Minkowski spacetime between geodesic motion and non-detection of quanta when the field is in the vacuum state, should be regarded as a very special result. In a generic static spacetime, one can define a vacuum in which any static detector is not excited. If the spacetime is ultrastatic, any static detector is also geodesic, so there is a state in which detectors belonging to one particular geodesic frame do not get excited. In the degenerate case of Minkowski spacetime, there are infinitely many such static frames, related to each other by Lorentz transformations, all defining the same vacuum, by Chmielowski's theorem 24. Together, these frames contain all the geodesic motions in Minkowski spacetime, and this explains why the detector remains in the ground state iff it moves along a geodesic. But this circumstance is accidental, and due to the fact that the high degree of symmetry of Minkowski spacetime allows one to identify the classes of static and geodesic frames. To generalise this, by claiming that the no-detection condition should always identify geodesic frames, is incorrect, as shown by the counter-examples of section 5 .

The same sort of degeneracy that we have in Minkowski spacetime also occurs in the Einstein static universe, as we saw in section 5.1. There, all frames rotating with a sufficiently small angular velocity correspond to a null response. This might be understood, heuristically, thinking of the particle detection, and of the corresponding field excitation, as a resonance phenomenon, ${ }^{11}$ an interpretation suggested by the form of the inequality (3.5). The quantity $\omega r / m$ is the phase speed, along the detector trajectory, of the field modes characterised by $\omega$ and $m .{ }^{12}$ For a discrete spectrum, there is a lower bound in $\omega / m$ (equal to $1 / a$ in the Einstein universe), so a detector whose angular velocity is below that limit fails to excite any mode and remains in its ground state. However, in an asymptotically flat spacetime the spectrum has a continuous part and the quantity $\omega / m$ can be arbitrary low, so a detector moving with any arbitrarily low speed can always excite a field mode. This removes the degeneracy mentioned above. In fact, the quantum vacuum in this case happens to be associated with a non-rotating frame.

The whole analysis of the present paper shows that the local behaviour of detectors in a curved background does differ from the one in Minkowski spacetime. It seems therefore, at first, that using particle detectors one can invalidate the equivalence principle. Such a conclusion is, however, incorrect. Einstein's equivalence principle applies only to experiments performed within a spacetime region $\mathcal{O}$ that satisfies two requirements:

(i) $\mathcal{O}$ is very small with respect to the typical scales associated with curvature;

(ii) The physical conditions of a non-gravitational nature in $\mathcal{O}$ are the same that one would

\footnotetext{
${ }^{11}$ We are grateful to Rickard Jonsson for pointing this out to us.

${ }^{12}$ Notice that this is not the same quantity as the component of the phase velocity along the trajectory. If $v_{\mathrm{ph}}^{i}$ are the coordinate components of the phase velocity ( $i$ and $j$ denote spatial indices, running from 1 to 3 ), and $k_{i}$ is the wave-number one-form, then the phase speed in the direction defined by the unit vector $e^{i}$ is $\left(k_{i} v_{\mathrm{ph}}^{i}\right) /\left(k_{j} e^{j}\right)$. The component of the phase velocity along the same direction is instead $h_{i j} e^{i} v_{\mathrm{ph}}^{j}$, where $h_{i j}$ is the metric of three-space.
} 
have within a similar region in Minkowski spacetime.

Since we consider point-like detectors, the detection process takes place locally, and requirement (i) is satisfied. However, the detector necessarily interacts with the field, which "feels" the entire spacetime through its quantum state - definitely a global concept. Hence, requirement (ii) does not apply, and local field observables in $\mathcal{O}$ behave, in general, differently than they would in a similar region of Minkowski spacetime. Although the detector behaviour is at variance with what one would expect on the basis of the equivalence principle, one cannot speak of a true violation, because the requirements under which the principle itself can be applied are not fulfilled. ${ }^{13}$ In short, it simply makes no sense to ask whether the particle detectors considered so far satisfy the equivalence principle.

There is a similarity between this issue and an old problem concerning the behaviour of electric charges in a gravitational field. The Lorentz-Dirac equation in a curved spacetime contains a term that accounts for possible effects of radiation backscattering off the curvature [25]. This also seems to violate the equivalence principle, but what is actually violated are, again, the very conditions for testing it, because the behaviour of a charge cannot be regarded as local, since it depends on its entire electromagnetic field. In particular, the radiation produced by the charge can be backscattered off the curvature, and affect the charge itself at later times. Thus, experiments involving radiation reaction, even if performed within a small neighbourhood of the charge - hence satisfying (i) - , are in general sensitive to the effects on it of curvature in regions that are far-away in spacetime. Therefore, such experiments do not meet requirement (ii). One can remove this objectionable feature by suitably adapting the experimental setting, though. For example, one can shield the charge enclosing it within a small metal box, thus preventing it from being affected by any radiation coming from distant regions - in particular, radiation generated through the backscattering of the charge's own field off spacetime curvature. The behaviour of the charge will then be the same as it would have been within a similar box in Minkowski spacetime. This should not appear as an implementation of the equivalence principle by brute force. Indeed, the very reason why the principle itself is interesting at all, is in order to check whether position invariance and Lorentz invariance still hold true, locally, in a curved spacetime [22]. Requirement (ii) is then necessary in order to exclude trivial violations of these properties.

Similarly, one can conceive experiments involving a particle detector in which requirement (ii) is also fulfilled. Enclose the detector within a perfectly reflecting box (i.e., impose the boundary condition $\phi \equiv 0$ on the walls of the box). The modes inside the box are now completely independent of the "external world," so the detector is, in fact, isolated from the field outside. In addition to this shielding, let us also assume that the coefficient $\xi$ in the field equation (2.1) is equal to $1 / 6$, so that no violation of the equivalence principle takes place already at the classical level 21. Then, the modes inside the box will be indistinguishable from those that one would have within a similar box in Minkowski spacetime, provided the box is sufficiently small. The response function will then be the same in the two cases [26]. Hence, it appears that Einstein's equivalence principle holds also for particle detectors, once requirement (ii) is duly fulfilled.

\footnotetext{
${ }^{13}$ See reference [5] for similar considerations about uniformly accelerated detectors.
} 


\section{Acknowledgements}

We wish to thank an anonymous board member for suggesting several improvements. SS is grateful to Antony Valentini for asking a question that stimulated this investigation, and to Tony Rothman for bringing reference [9] to his attention. He also gratefully acknowledges warm hospitality from the Department of Astronomy and Astrophysics at Chalmers University, where part of this work was done.

\section{References}

[1] N. D. Birrell and P. C. W. Davies, Quantum Fields in Curved Space (Cambridge, Cambridge University Press, 1982).

[2] L. Smolin, "On the nature of quantum fluctuations and their relation to gravitation and the principle of inertia," Class. Quantum Grav. 3, 347-359 (1986).

[3] S. A. Fulling, "Nonuniqueness of canonical field quantization in Riemannian space-time," Phys. Rev. D 7, 2850-2862 (1973).

[4] J. R. Letaw and J. D. Pfautsch, "Quantized scalar field in the stationary coordinate systems of flat spacetime," Phys. Rev. D 24, 1491-1498 (1981).

[5] L. P. Grishchuk, Ya. B. Zel'dovich, and L. V. Rozhanskiur, "Equivalence principle and zeropoint fluctuations," Sov. Phys. JETP 65, 11-14 (1987) [Zh. Eksp. Teor. Fiz. 92, 20-27 (1987)].

V. L. Ginzburg and V. P. Frolov, "Vacuum in a homogeneous gravitational field and excitation of a uniformly accelerated detector," Sov. Phys. Usp. 30, 1073-1095 (1988) [Usp. Fiz. Nauk 153, 633-674 (1987)].

[6] P. G. Grove and A. C. Ottewill, "Notes on 'particle detectors'," J. Phys. A 16, 3905-3920 (1983).

[7] S. A. Fulling, "Alternative vacuum states in static space-times with horizons," J. Phys. A 10, 917-951 (1977).

[8] J. R. Letaw and J. D. Pfautsch, "Quantized scalar field in rotating coordinates," Phys. Rev. D 22, 1345-1351 (1980).

J. R. Letaw, "Stationary world lines and the vacuum excitation of noninertial detectors," ibid. 23, 1709-1714 (1981).

[9] P. C. W. Davies, T. Dray, and C. A. Manogue, "Detecting the rotating quantum vacuum," Phys. Rev. D 53, 4382-4387 (1996) gr-qc/9601034.

[10] V. A. De Lorenci and N. F. Svaiter, "A rotating quantum vacuum," Found. Phys. 29, 12331264 (1999) hep-th/9612006.

V. A. De Lorenci, R. D. M. De Paola, and N. F. Svaiter, "The rotating detector and vacuum fluctuations," Class. Quantum Grav. 17, 4241-4253 (2000) hep-th/0005171;

Erratum: ibid. 18, 205 (2001).

R. D. De Paola and N. F. Svaiter, "A rotating vacuum and a quantum version of Newton's bucket experiment," Class. Quantum Grav. 18, 1799-1808 (2001) ["A rotating vacuum and the quantum Mach's principle," gr-qc/0009058. 
N. Nicolaevici, "Null response of uniformly rotating Unruh detectors in bounded regions," Class. Quantum Grav. 18, 5407-5411 (2001).

L. Sriramkumar and T. Padmanabhan, "Probes of the vacuum structure of quantum fields in classical backgrounds," Int. J. Mod. Phys. D 11, 1-34 (2002) gr-qc/9903054.

[11] J. S. Bell and J. M. Leinaas, "Electrons as accelerated thermometers," Nucl. Phys. B 212, 131-150 (1983).

O. Levin, Y. Peleg, and A. Peres, "Unruh effect for circular motion in a cavity," J. Phys. A 26, 3001-3011 (1993).

[12] R. M. Wald, General Relativity (Chicago, University of Chicago Press, 1984).

[13] D. W. Sciama, P. Candelas, and D. Deutsch, "Quantum field theory, horizons and thermodynamics," Adv. Phys. 30, 327-366 (1981).

W. G. Unruh and R. M. Wald, "What happens when an accelerating observer detects a Rindler particle," Phys. Rev. D 29, 1047-1056 (1984).

T. Padmanabhan, "Why does an accelerated detector click?" Class. Quantum Grav. 2, 117126 (1985).

P. G. Grove, "On an inertial observer's interpretation of the detection of radiation by linearly accelerated particle detectors," Class. Quantum Grav. 3, 801-809 (1986).

D. J. Raine, D. W. Sciama, and P. G. Grove, "Does a uniformly accelerated quantum oscillator radiate?" Proc. R. Soc. London A 435, 205-215 (1991).

W. G. Unruh, "Thermal bath and decoherence of Rindler spacetimes," Phys. Rev. D 46, 3271-3277 (1992).

B. F. Svaiter and N. F. Svaiter, "Inertial and noninertial particle detectors and vacuum fluctuations," Phys. Rev. D 46, 5267-5277 (1992);

Erratum: ibid. 47, 4802 (1993).

G. E. Matsas, "Rindler and Minkowski particles relationship revisited," Phys. Lett. B 380, 24-28 (1996) gr-qc/9605009.

B. L. Hu and A. Raval, "Is there emitted radiation in the Unruh effect?" in Quantum Aspects of Beam Physics, edited by P. Chen (Singapore, World Scientific, 2002), pp. 414-423 quant-ph/0012134.

[14] V. P. Frolov and I. D. Novikov, Black Hole Physics (Dordrecht, Kluwer, 1998), pp. 364-372.

[15] D. G. Boulware, "Quantum field theory in Schwarzschild and Rindler spaces," Phys. Rev. D 11, 1404-1423 (1975).

[16] G. B. Arfken and H. J. Weber, Mathematical Methods for Physicists, 4th ed. (San Diego, Academic Press, 1995), p. 738.

[17] S. Sonego and M. Massar, "On the notions of gravitational and centrifugal force in static spherically symmetric space-times," Mon. Not. R. Astron. Soc. 281, 659-665 (1996).

S. Kristiansson, S. Sonego, and M. A. Abramowicz, "Optical space of the Reissner-Nordström solutions," Gen. Relativ. Gravit. 30, 275-288 (1998).

[18] P. M. Morse and H. Feshbach, Methods of Theoretical Physics, part I (Boston, McGraw-Hill, 1953), pp. 766-768.

K. Gottfried, Quantum Mechanics, vol. I (New York, Benjamin, 1966), pp. 48-50.

[19] M. A. Abramowicz, J. C. Miller, and Z. Stuchlík, "Concept of radius of gyration in general relativity," Phys. Rev. D 47, 1440-1447 (1993). 
[20] M. Abramowitz and I. A. Stegun, Handbook of Mathematical Functions (New York, Dover, 1972), p. 334, eq. 8.6.1.

[21] S. Sonego and V. Faraoni, "Coupling to the curvature for a scalar field from the equivalence principle," Class. Quantum Grav. 10, 1185-1187 (1993).

V. Faraoni and S. Sonego, "The equivalence principle determines the coupling constant to the curvature for a scalar field," in Proceedings of the 5th Canadian Conference on General Relativity and Relativistic Astrophysics, edited by R. B. Mann and R. G. McLenaghan (Singapore, World Scientific, 1994), pp. 386-390.

A. A. Grib and E. A. Poberii, "On the difference between conformal and minimal couplings in general relativity," Helv. Phys. Acta 68, 380-395 (1995).

[22] C. M. Will, Theory and Experiment in Gravitational Physics (Cambridge, Cambridge University Press, 1993).

[23] L. H. Ford, "Quantum vacuum energy in a closed universe," Phys. Rev. D 14, 3304-3313 (1976).

[24] P. Chmielowski, "States of scalar field on spacetimes with two isometries with timelike orbits," Class. Quantum Grav. 11, 41-56 (1994).

[25] B. S. DeWitt and R. W. Brehme, "Radiation damping in a gravitational field," Ann. Phys. (N.Y.) 9, 220-259 (1960).

C. Morette DeWitt and B. S. DeWitt, "Falling charges," Physica 1, 3-20 (1964).

[26] O. Levin, Y. Peleg, and A. Peres, "Quantum detector in an accelerated cavity," J. Phys. A 25, 6471-6481 (1992). 